\title{
MODIFIED DC-DC CONVERTER FOR HIGH INPUT VOLTAGE APPLICATIONS
}

\author{
Battula.VeeraVasanthaRao ${ }^{1}$, Sravanthi.Kantamaneni $^{2}$ \\ ${ }^{1}$ Assistant Professor, E.E.E Dept., ${ }^{2}$ Assistant Professor, E.C.E Dept \\ veeravasantharao@gmail.com, ${ }^{2}$ sravanthi.kantamneni@yahoo.com
}

\begin{abstract}
:
This paper proposes a model for high input voltage applications using two fly-back converters. In this the components are arranged by using integrated magnetic techniques. To reduce the voltage stresses the input components are connected in series and to obtain the required output the output components are connected in parallel. The three cores of the fly-back transformers are air gaped to reduce the current spikes which are caused due to coupling and the secondary side of the transformers are inversely coupled for ripple cancellation. This is done by using a prototype which helps us to obtain output as we desired for certain input with certain transformation ratio. The components used are IGBT's, capacitors and fly-back transformers.
\end{abstract}

Keywords: Integrated magnetic techniques fly back converter, coupling, ripple reduction, interleaved operation. $* * * * *$

\section{INTRODUCTION:}

Integrated magnetic techniques combine transformers and inductors into a single core in order to reduce the size and cost of magnetic components in $\mathrm{dc} / \mathrm{dc}$ converters. Principles of modeling and designing integrated magnetic have been widely published. Historically, integrated dc/dc converters were first developed for Forward converters. However, recently integrated magnetic full wave circuits have been extensively studied. These full wave circuits have reported significant benefits, including high efficiency, high power density and low switch stress. Applications of these circuits are being proposed in $30 \mathrm{~W}-100 \mathrm{~W}$ ranges, and have great potential for telecommunication power supplies. A "typical"2 integrated magnetic full wave dc/dc converter, which uses a three-leg magnetic core. This class of full wave integrated magnetic circuit's exhibits a common character that there is no winding on the inductor leg. Instead, the secondary windings play the role of the equivalent Inductor winding, and hence, the secondary windings must satisfy both power transfer and current ripple restrictions for different operating stages. The elimination of the inductor winding comes from the magnetic integration methods, and, in applications, this is believed to reduce the copper loss and simplify the transformer structure. However, optimum transformer design must keep the balance between copper loss, ferrite loss and total size. In integrated magnetic transformers, the number of secondary winding turns influences the number of primary winding turns, the magnetic core size and the output current characteristics. This places a burden on the secondary winding turn decision and complicates the transformer design. As a result, the magnetic core often needs to be specially designed and manufactured in order to satisfy both output current ripple requirements and flux density constraints. Using flyback converter this integrated magnetic techniques are employed.It is equivalent to that of a buck-boost converter, with the inductor split to form a transformer. Therefore the operating principle of both converters is very close:

When the switch is on, the primary of the transformer is directly connected to the input voltage source. This results in an increase of magnetic flux in the transformer. The voltage across the secondary winding is negative, so the diode is reverse-biased (i.e., blocked). The output capacitor supplies energy to the output load. When the switch is off, the energy stored in the transformer is transferred to the output of the converter. The operation of storing energy in the transformer before transferring to the output of the converter allows the topology to easily generate multiple outputs with little additional circuitry, although the output voltages have to be able to match each other through the turns ratio.

This paper uses two fly-back converters which act as fly-back transformers which are designed under a single magnetic core. Earlier they designed a converter based on this concept. The main features of that converter are

- Earlier designed a single magnetic core three limbed transformer with center limb air gapped. So that the current spikes can be reduced.

- The primary and secondary side of the transformer is connected in parallelthe operation is similar to that of a fly-back converter with two switches on the primary side and two capacitors on the secondary side. The secondary side is directly coupled. 
Whereas coming to the modified converter the changes made and the specified reasons for the changes are as discussed below.

- Connecting the primary side in series reduces the voltage stresses of the primary components. The primary switches are rated at half of the corresponding voltage values in a single Fly-back or a traditional full wave buck-boost power converter.

- The on-resistance and cost of the primary switches are, thus, significantly reduced. Alternatively, this implies that for the same rated switches, the proposed converter can handle twice the input voltage compared with the converter.

- Interleaved operation with paralleled connection on the secondary side keeps the benefits of ripple reduction as a traditional full wave buck-boost power converter does.

- To satisfy a certain current ripple requirement, magnetizing inductance can be reduced by means of suitable inductor coupling design.

- Due to the mutual influence of the two coupled inductors that is combined with the transformers, further current ripple reduction can be achieved when compared with interleaved Fly-back converters without coupling.

- Also, the amount of the magnetic components is reduced by integrating the two transformers into one magnetic core, although the efficiency of the new approach may not be improved when compared with conventional discrete interleaved Fly-backs.

- Current spike caused by the voltage mismatch between the windings can be suppressed by gapping all the center and outer legs. Compared with tight coupling, such as the condition in a traditional full wave buck-boostpower converter, gapping all the legs weakens the coupling between the two Fly-backs' windings, and thus, reduces the effect of the voltage mismatch. Between the two Flybacks' windings, and thus, reduces the effect of the voltage mismatch.

The modified converter is as shown below which consists of IGBT's which act as switches on the primary side, thecapacitors help in the timing operation of the converter depending on the intervals. And the secondary consists of diodes D1 and D2 which are reversely coupled for the sake of ripple reduction. And the operation of the converter is as discussed below.

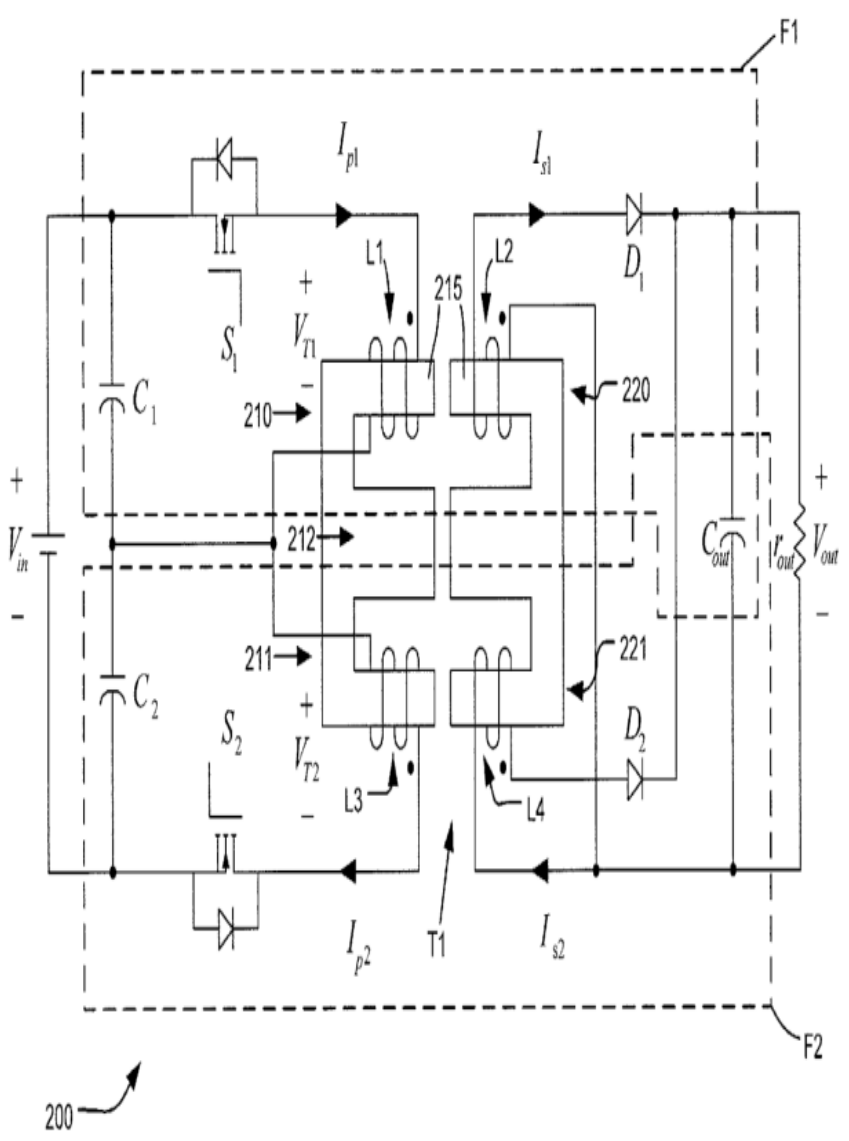

Figure representing the circuit model of the modified dc-dc converter Operation principle

The main primaries switch and are the auxiliary switches, which are connected with the reset windings to obtain active clamp. Vc1, Vc2, Vc3 are the voltages across c1, c2, c3. Capacitors $\mathrm{Cr} 1$ and $\mathrm{Cr} 2$ are the equivalent resonant capacitors of $\mathrm{q} 1, \mathrm{q} 2 . \mathrm{c} 1, \mathrm{q} 1, \mathrm{t} 1, \mathrm{q} 3$ and $\mathrm{c} 3$ are the primary side components of one active-clamp forward, while the other forward consists of $\mathrm{c} 2, \mathrm{q} 2, \mathrm{t} 2, \mathrm{q} 4$ and $\mathrm{c} 3$. The two forward circuits share the clamping capacitor $\mathrm{c} 3$ so that the charge balances of $\mathrm{c} 1$ and $\mathrm{c} 2$ are kept. Since the control signals of the two channels have the same duty cycle, unbalanced voltage on c1, c2 and can generate different magnetizing current to charge the capacitors. Therefore, the two input voltages are adjusted to a balanced value at steady state. Also, the two channels share the freewheeling switch q7 and output inductor L1on the secondary side. Therefore, there is no current sharing problem.

The size of the output inductor is reduced due to interleaved operation. One potential concern is the effect of the leakage inductor between the primary winding and the active clamp winding. The energy stored in the leakage inductor can impose excessive voltage spikes on the switches. This leads to higher voltage stress on the switches and should be taken into 
account for design. Thus, transformers with tight coupling are required. Also, effective snubber is helpful to reduce the voltage stress. The following description explains the detailed operation principle of the proposed topology.

In general, circuit operation can be qualitatively described over several modes/intervals. The upper forward circuit transfers energy to secondary side during time interval t0 $\mathrm{t} 1$. The under forward circuit transfer energy during interval $\mathrm{t} 7$ $-\mathrm{t} 8$. Interval $\mathrm{t} 4-\mathrm{t} 5$ and interval $\mathrm{t} 11-\mathrm{t} 12$ are utilized for the freewheeling process of theoutput current. All the remaining intervalsare transition intervals and are substantially smaller in length than energy transfer or freewheeling time intervals. Interval $\mathrm{t} 0-\mathrm{t} 14$ is defined as the switching period $\mathrm{T} 1$. The lengths of $\mathrm{t} 0-\mathrm{t} 1$ and $\mathrm{t} 7-\mathrm{t} 8$ are defined as DT, which represents the duty ratio, and thus, the duty ratio $\mathrm{D}$ will always be less than one-half. The lengths of $t 1-t 4$ and $t 8-t 11$ are set to a suitable value td, which is longer than the required transition time $\mathrm{t} 1-\mathrm{t} 3$ and $\mathrm{t} 8-\mathrm{t} 10$. The length of $\mathrm{t} 0-\mathrm{t} 1$ and $\mathrm{t} 7$ $-\mathrm{t} 8$ are defined as DT, which represents the duty ratio.

\section{FLUX DISTRIBUTIONS ANALYSIS:}

For the convenience of magnetic design, it is necessary to providethe formulas for flux distributions. To prevent the magneticcore from saturating, the peak values of the flux densities in allthree legs should be keptbelow a specified maximum valueTherefore, this section will discuss the detailed calculation forboth the ac fluxes and dc fluxes in all three legs. As a result, thepeak values of the fluxes are the sum of the dc fluxes and half of the ac fluxes showsthe magnetic reluctance circuit of the proposedtopology, assuming the conditions of the two outer legs are symmetrical. Also, Fig shows the flux path of in the core of transformer.Because of the air gap, the center leg is no longer alow magnetic reluctance path for the fluxes. The flux generated by each winding in the outer legs can go through all threelegs. The flux interaction between the windings affects the currentripple and peak values of the flux densities Furthermore, following figure shows the detailed equivalent magneticreluctance circuit during each interval. The effect of the correspondingwinding is removed when the current in the windingis zero.

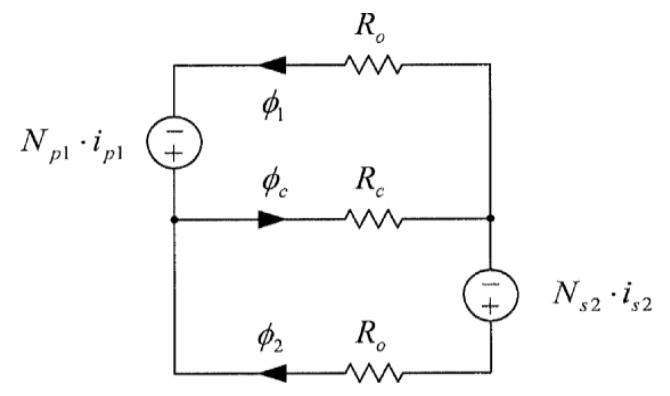

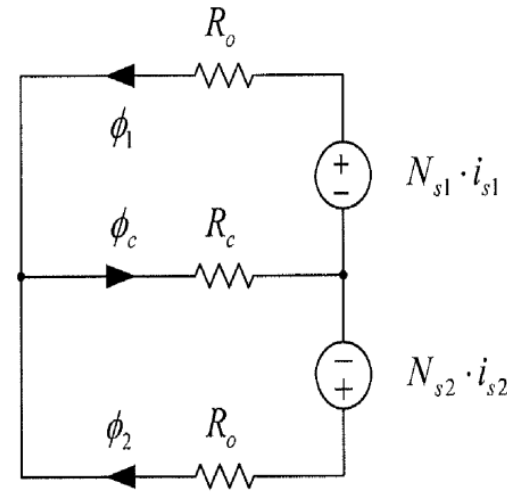
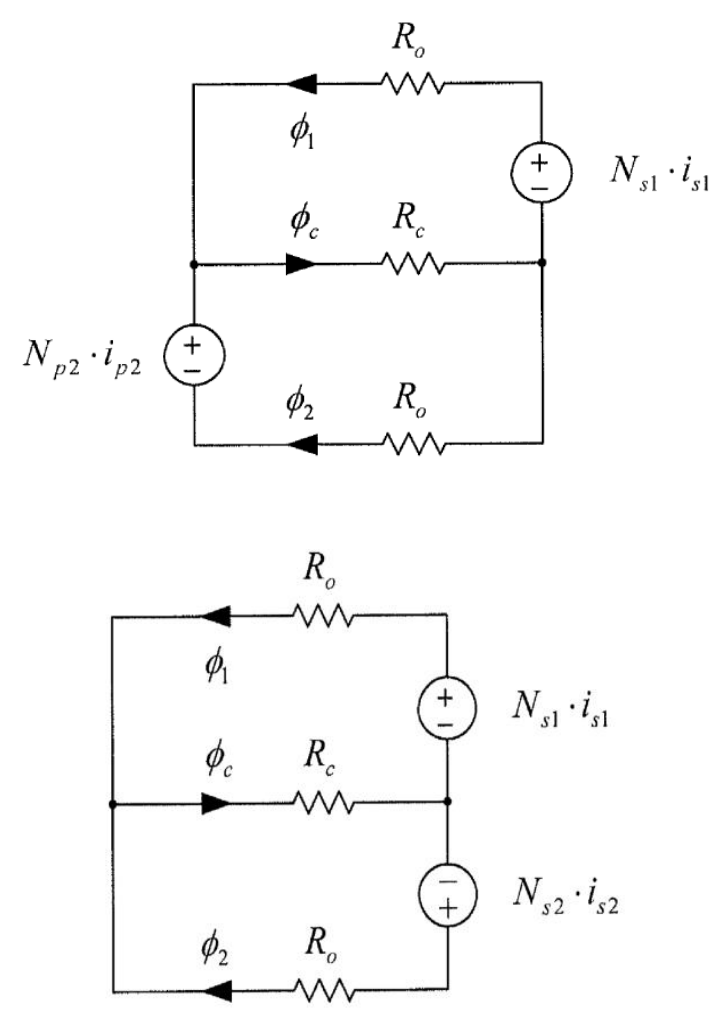

Figures a,b,c,d respectively shows the flux path in the core

Of which fig a represents flux path during the interval t0-t1 similarlyfig $\mathrm{b}$ during $\mathrm{t} 1 \mathrm{-t} 2$, fig $\mathrm{c}$ during $\mathrm{t} 2-\mathrm{t} 3$ and fig d during t3-t4. The equations corresponding to their flux paths and intervals are

$$
\begin{aligned}
& \text { T0-T1: } \Phi \mathrm{c}=(\mathrm{Vin} / 2 \mathrm{~Np})-(\mathrm{V} 0 / \mathrm{Ns}) \\
& \text { T1-T2: } \Phi \mathrm{c}=-(2 \mathrm{~V} 0 / \mathrm{Ns}) \\
& \text { T2-T3: } \Phi \mathrm{c}=(\mathrm{Vin} / 2 \mathrm{~Np})-(\mathrm{V} 0 / \mathrm{Ns}) \\
& \text { T3-T4: } \Phi \mathrm{c}=-(2 \mathrm{~V} 0 / \mathrm{Ns})
\end{aligned}
$$

Thus by the analysis all the fluxes in the system i.e. both the ac and dc fluxes obtained are 
Total ac flux in the two outer limbs is obtained as

$$
\begin{aligned}
& \Delta \phi 1=\Delta \phi 2=\mathrm{V} 0(1-\mathrm{D}) / \mathrm{Nsfs} \\
& \text { The total ac flux in the center limb obtained as } \\
& \Delta \phi \mathrm{c}=\mathrm{V} 0(1-2 \mathrm{D}) / \mathrm{Nsfs} \\
& \text { DC Flux: } \\
& \Phi \mathrm{c}=\phi 1+\phi 2 \\
& \quad=(\mathrm{Np} 1 \mathrm{Ip} 1+\mathrm{Ns} 1 \mathrm{Is} 1+\mathrm{Np} 2 \mathrm{Ip} 2+\mathrm{Ns} 2 \mathrm{Is} 2)(1 /(\mathrm{R} 0+2 \mathrm{Rc})
\end{aligned}
$$

To avoid the saturation of the magnetic core, the peak value ofthe flux densities of all three legs should be computed. The peak value can be obtained by the sum of DC fluxes and half of the total AC fluxes. By using the calculation of the current ripple and the peakflux densities suitable magnetic core size and the turnnumbers of the windings can be chosen to satisfy the currentripple restrictions and the magnetic constraints.

\section{SIMULATION AND RESULTS:}

The Simulink model of the converter is as shown below. In this dual converter two interleaved forward circuits are connected in series on the primary side and in parallel on the secondary side the two transformers share one low voltage capacitor for clamping through auxiliary windings and switches. Through the resonance between the magnetizing inductors and equivalent capacitors of the switches during the transition time, switching loss of the primary switches can be reduced. Output current and voltage wave forms when firing pulses from same firing circuit. When firing pulses are giving from a single pulse generating circuit wave forms are shown in graph here input voltage is $400 \mathrm{~V}$, output is $20 \mathrm{~V}$ and current.

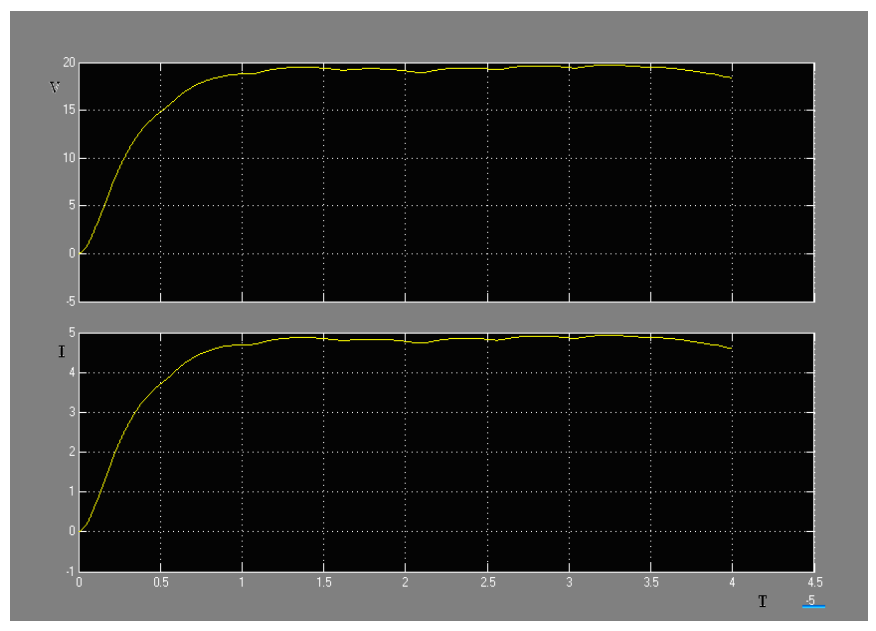

Fig-1: output current and voltage wave forms when firing pulses from same firing circuit.

When firing pulses for individual switches is given from different firing circuits the only difference is output voltage, output current values are changed and in this input voltage is same is same that is $400 \mathrm{~V}$.but output voltage is $25 \mathrm{~V}$ and output current is $0.7 \mathrm{~A}$

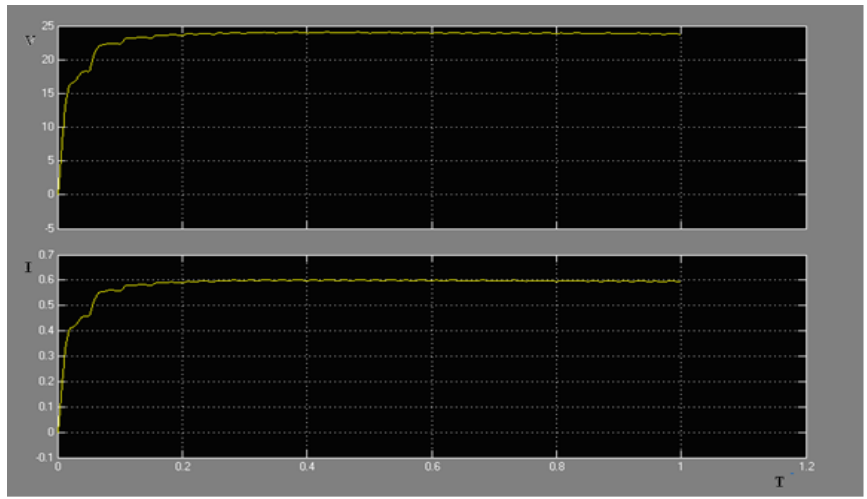

Fig-2: output voltage and current wave forms when firing pulses are from different firing circuits.

Specifically, the following differences are remarked when compared with a traditional converter in.

1) The new approach connects the input of the two Fly backs in series, while the traditional method connects the input in parallel.

2) The proposed converter has three gaps in all legs. The traditional converter only has one gap in the center leg.

3) The secondary windings are inversely coupled in the new converter, and directly coupled in the traditional converter.

From the first difference, it can be concluded that the proposed method has half of the voltage stress when compared with the traditional method. According to the second and third differences, the secondary windings of the traditional converter are directly coupled with tight coupling. However, for practicalcircuits, especially for this high voltage application, switches cannot operate exactly simultaneously. There is a voltage mismatch between the two secondary windings. The voltage difference is applied to the leakage inductance between the twowindings. Because the two outer legs have low reluctances, and the center leg has a high reluctance, the two secondary windings have good coupling and small leakage inductance. Minor mismatch can lead to high current spikes and resonance. On the other hand, the secondary windings of the new converterare inversely coupled with not so tight couplingsince all three legs are gapped. By gapping all the three legs, a big leakage inductance between the windings of the two

Transformers is obtained, since the flux generated by each winding in the outer legs can go through all three legs (is approximately equal to $1 / 3$ when all the legs have gaps with approximately same distances.) The large inductance value can avoid high current spike when a voltage mismatch is applied. Also, current ripple reduction can still be achieved with suitable coupling design. We also remark that the weakened coupling between the two transformers allows the duty ratio to be greater than $50 \%$ in the propose circuit 


\section{CONCLUSIONS}

The dual interleaved forward converter may be beneficial for high current/ high power density designs. This converter topology would be ideal for intermediate bus converters and merchant power applications because the reduced input and output capacitor ripple current reduces electrical stress on the input and output capacitors. The inductor ripple current cancellation of interleaving converters allows the design more output capacitance, which in turn should reduce the design's output capacitance requirements.

Interleaving techniques are adopted to reduce input/output current or voltage ripples for converters with critical conduction mode. This is useful for not only buck operation and also for boosting purpose. In this study implements two types of phase-shift control schemes for critical interleaved boost PFC converters. Sub-harmonic oscillation phenomena occur at high line voltage when one of them is adopted. So this paper presents a quantitative analysis on the phenomena and proposes a novel phase-shift control scheme.

\section{REFERENCES:}

[1] G. C. Hua and F. C. Lee, "Novel zero voltage transition PWM converters,' in Proc. IEEE Power Electronspec.conf., 1992, pp.55-61.

[2] J.G. Cho et al., "zero-voltage and zero-current-switching full bridge PWM converter for high-power applications,' IEEE Trans. Power Electron., vol.11, pp.622-628, July 1996.

[3] T. Morimoto, shirakawa, O. Koudriavtsev, and M. Nakaoka, ' zero-voltage zero current hybrid soft-switching phase-shifted PWM dc-dc power converter for high power applications, 'in Proc.IEEE APEC'00,New Orleans,LA,Feb.2000,pp.104-110.

[4] R. Redl, N.O. Sokal, and L. Balogh, " A novel softswitching full-bridge $\mathrm{dc} / \mathrm{dc}$ converter: analysis, design considerations, and experimental results at $1.5 \mathrm{KW}, 100 \mathrm{KHz}$, " IEEE Trans.Power Electron., vol.6,pp.408-418, july 1996.

[5] H.J.Kim and H.J.Kim, "Active clamp forward converter with MOSFET synchronous rectification," in Proc. Power Electronics Specialists conf., 1994, vol.2.pp.895-901.

[6] Y.K.Lo, T.S.Kao, and J.Y.Lin, "Analysis and design of an interleaved active-clamping forwardconverter, IEEEtRANS.lnd.Electron" vol.54, no.4, pp.2323-2332, Aug 2007. 